\title{
Monitoring of Sona Massori Paddy Crop and its Pests Using Image Processing
}

\author{
Prof. Barry Wiling \\ Professor, Department of Computer Science, U.S.M.N Oman \\ barrywiling@usmn.edu
}

\begin{tabular}{|l|l|}
\hline Article History & \multicolumn{1}{|c|}{ Abstract } \\
$\begin{array}{l}\text { Article Submission } \\
19 \text { February 2017 } \\
\text { Revised Submission } \\
12 \text { April } 2017 \\
\text { Article Accepted } \\
13 \text { May 2017 } \\
\text { Article Published }\end{array}$ & $\begin{array}{l}\text { Nowadays plant diseases are the major cause of low agriculture yield. So } \\
\text { significance of detecting diseases in early stages and treating it will improve the } \\
\text { agriculture yield. In India the major agriculture crop is paddy and in central part of } \\
\text { south India there is a specific paddy crop called Sona Massori. In our work we } \\
\text { concentrated on Sona Masori paddy crop health and pest monitoring using image } \\
\text { processing. Here image processing technique is used to observe the image of the leaf } \\
\text { and based on the image the diseases are identified using the following process such } \\
\text { as image acquisition, pre-processing, segmentation, feature extraction and } \\
\text { classification. Mostly diseases are caused by pests, insects and pathogens. In Sona } \\
\text { Massori paddy crop 99\% of diseases are caused by pests, So detecting pests also } \\
\text { plays an important role in improving yield. Here pests are detected by image } \\
\text { processing techniques such as Gaussian blur and morphological operations. } \\
\text { Keywords: Image Processing, Diseases, Object Extraction, Paddy crop, } \\
\text { Segmentation }\end{array}$ \\
\hline
\end{tabular}

\section{Introduction}

In India timely diagnosis of plant diseases is an important task because of change in climatic conditions. Because non-identification of diseases will lead to low yield. Most used method for Sona Massori paddy plant disease detection is naked eye observation. For large field naked eye observation is impossible due to lack of human resource. So the evolution of automatic detection is emerged to detect diseases with less timing and less cost. In plants the diseases affected area is detected and measured with image processing technique. Diseases affected area is determined with change in the color [1]. Segmentation will separate captured image into various essential parts. Here we concentrated on various methods to perform segmentation, range from specified threshold process to advanced many color segmentation process.

Thickness of various pests in Sona Massori paddy fields is very significant for the pest prediction decision. Generally old technique trap method is used to catch the pests. The attentive insects are brought to the testing lab to detect manually. Typically paddy field technicians recognize and then isolate the pests physically according to their species [2]. The resultant output is used to approximation the insect thickness in the Sona Massori fields. Nevertheless, many site and recurrent including of paddy insects is time waste and tough process. It can direct to very less count correctness and delay in obtain absolute count.

Digital imaging processing technique made easy to detect pests and to monitor the health of the plant automatically. Image processing technique which worn for measure exaggerated disease area, and it is decides the dissimilarity in shade of the area which is affected. Image segmentation is a popular procedure of unraveling or federation of picture into diverse parts [3]. As we know there are many different ways of the stage segmentation, range for the easy threshold method to higher color segmentation method. The segmentation progression is base on facial appearance available in the paddy picture. This color in order, limitations of segment of a picture. 


\section{Related Work}

Widespread experiments have been conduct in order to discover methods for detection of plant diseases automatically. Many diseases apparently in plants such as main roots, full stem, seed or leaf buds. Early it was declared that work concentrates mostly on leaves.[4] describes the tactic for appreciation of diseases in plant here on seed and fruit. This work is collected of K-Means technique and the pictures are confidential by machine learning. They urban a method for detect the illustration signs of plant diseases by with the image processing algorithm. The correctness of specified algorithm is experienced compare the pictures, which were segmented physically persons automatically segmented. The various processes for segment the disease division of paddy plant. This work too discuss about trait extraction technique to extract points of infected leaves and categorization of paddy diseases [5].

ANN method used in organization of disease in sona massori paddy which as organizing map, propagation method, and SVMs will be resourcefully done. At first input picture is in use and then processing is in progress. Environment and grey pixels together segmented in initial step. Huge and multiplication fraction of picture is also estranged. Finally small part and diseases area and its $\%$ and biological sculpture of disease is acquire that is major work with the discussed methodology [6]. The objective of current research is to supply the progress and improvement in calculating classification of back propagation approach.

Easy method was worn by the cultivators on the way to attain their objective since they intend to get, numbers or examine a little fraction of insects inhabitants [7]. They really show the trend in crowd of being. This method has 2 basic rules to experiment and the insect administration decisions, which usually require a substantial range, hard work and economy. In recent research few sampling techniques used for insect management like illuminant trap method, that creates unreliable sample which has bad quality for find difference cyclic and weekly catch of pests, but results are subject in change over pests actions and catch not any flying pests[8].As we know sweep net method is a fast and very cheap and effective for example arthropods living in awning of on Sona massori rice. But they have human being error because of inconsistency and poor hold of insects at the value point of sona massori plant; beating the rice, this is only sampling way that exploit a collect paddy plant group explanation or fertilizer with chemicals to destroy the whole rice crop[9].Comparatively less researched grassland in pe vision is 3-d change alteration. Previous approach used physically made 3-d website model to form correspondence between images so that alteration detection algorithm rule is practical. For this sort of come near to be utilize in modern application and consequently the overhead of construct 3-d geometry is not viable.

After catching, unwanted things are identified and rapidly destroy in the paddy crop that including and required data observation will be easy to hold on trap , it events butterflies movement and migration will grasp non flying pests[10]. By the way yellow trap is also cheap and it tracks pests migration, high categorization and including of samples, the repulsion is because of color incentive and do not hold not any flying insects. It is concluded that image processing techniques provides better results and it is very economic in detecting diseases and pests in sona massori rice plants.

\section{Proposed Method}

In our work first diseases in the plant is detected using Image processing technique. Here totally 5 steps are involved in finding out the diseases. These steps are as follows: Image Acquisition, Pre-Processing, Segmentation, Feature Extraction, then finally Detection and Classification of sona massori paddy plant disease. The possible infections in the sona massori rice plant will be identified and classified. In first step the imagery of sona massori leaf are captured by the tilt digital automatic camera. This captured image is in three colour Red- Green - Blue format. Color alteration nature for the sona massori RGB paddy leaf picture is bent, and instrument nondependent space color renovation for transformation of color organization is functional. In second step involves in removing noise in the captured picture. In third step i.e. pre-processing technique 
cropping of the paddy leaves picture will get picture area. Image enhancement process is then done to increase the contrast. Then Red-Green-Blue pictures are splinted into the grey images mathematical expression

$$
\mathrm{z}(\mathrm{a})=0.2342 * \mathrm{R}+0.6523 * \mathrm{G}+0.9852 * \mathrm{~B}
$$

Then to enhance sona massori plant disease, image equalization method is used. Cumulative distribution calculation is worn to allocate intensified high values. In third step image segmentation will be done. That is Partition of image into a range of part of identical features or having a number of similarities. This can be through using different methods like Otsu, k-means clustering, converting RGB TO HSI model etc. Among all these stated techniques Otsu clustering method will provide best accuracy. K-means clustering will worn for categorization of features based on different object set into $\mathrm{K}$ numbers. The categorization of objects are completed by minimize the length between object and corresponding cluster by squaring the sum.

\section{OTSU Threshold Algorithm:}

a) Based on threshold pixels are divided into 2 clusters.

b) Finding mean value of each divided cluster.

c) Difference between the mean values will be squared.

d) Increase the number of pixels in one cluster. The affected sona massori paddy leaf details of the diseases symptoms.

Extraction of features of plant plays a vital part in recognition of an image. In many applications of image processing techniques extraction is worn. Color and morphology methods are the sources in detection of diseases in sona massori plant. Captured picture is converted to HSI by the following postulate.

$$
M=\{\text { Beta if } R<B \text { 180-Beta if } G>R\} X=1-2 /\left(R^{*} G * B\right) Y=(1 / 2)(R * G * B)
$$

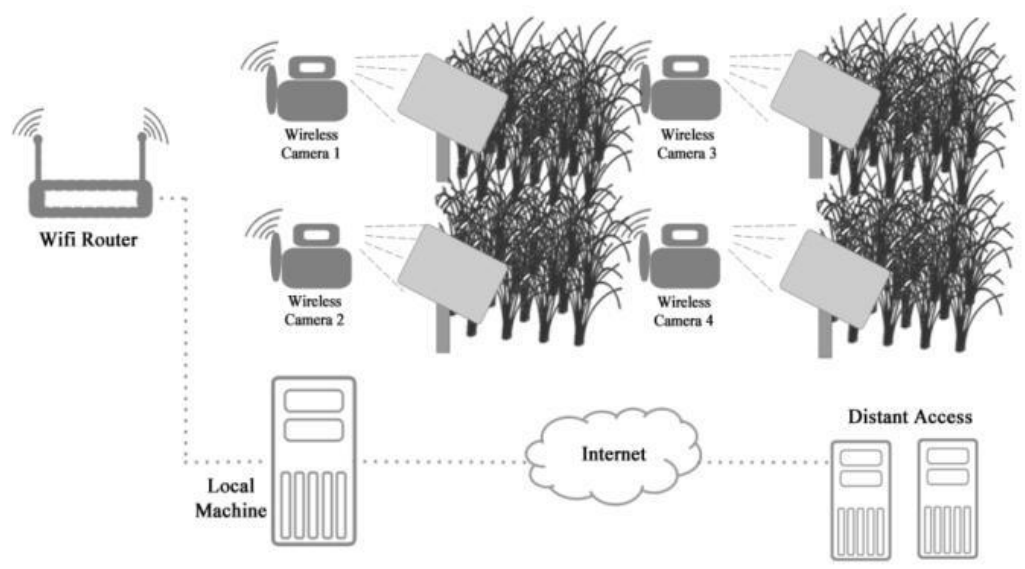

Fig 1: Pest detection process

\section{Simulation Results}

The simulations are performed using MATLAB. To distinguish between paddy leaves and non-paddy leaf part, $\mathrm{M}$ and Y solutions from HSI and segmentation color space are considered. The detection of diseases in paddy is simulated by MATLAB simulation tool and outputs are shown in Figure 2 and Figure 3 respectively 




Fig 2: Disease detected leaf

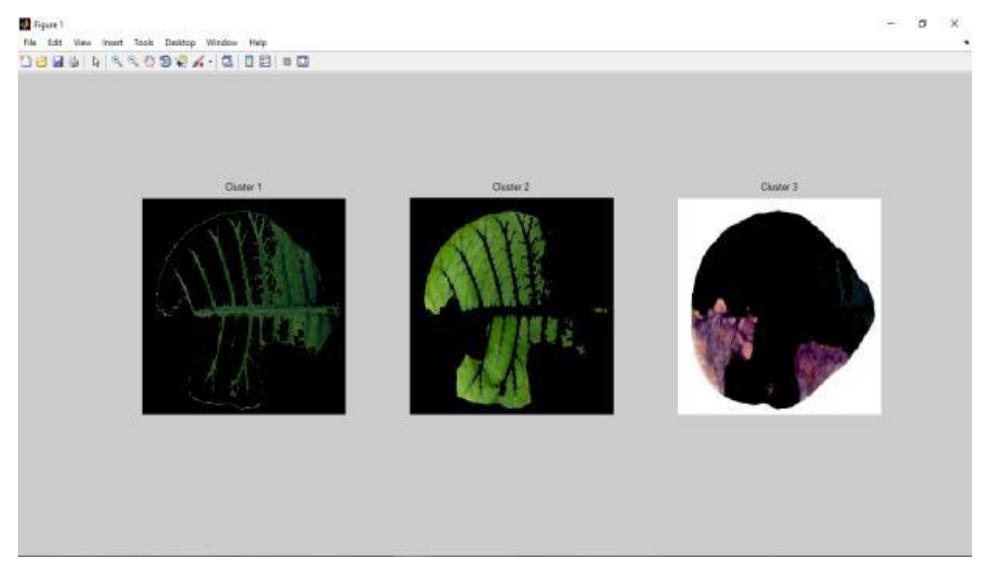

Fig 3: Process of detecting disease in the leaf

Next our work concentrates on detection of pests in sona massori paddy crop. The specified method used to identify the unwanted pests in the paddy picture is easy and still competent. Pixel values of the succeeding captured picture from the digital camera. Both the pictures are used in identified the dissimilarity. The initial image used as a reference image that presents the pixel values for comparing purpose, then the second image act as the input image. These two images were compared and pixel values were determined. If the inputted image pixel values are not equal to the reference image pixel values, then the input picture pixel will be used as an output image pixel as shown in Figure 3. If suppose the pixel value of the considered input picture is equal to the second image then the color of the background will be white. To conclude the difference of the reference and input image, the following postulate will be used:

$$
O_{R I} \mathrm{a}, \mathrm{b}=255, \text { if } \operatorname{Red}(\mathrm{a}, \mathrm{b})=I \mathrm{a}, \mathrm{b}
$$

$I \mathrm{a}, \mathrm{b}$, if $R(\mathrm{a}, \mathrm{b}) \neq I(\mathrm{a}, \mathrm{b})$ where $I(\mathrm{a}, b)$ is the input image and $\mathrm{R}(\mathrm{a}, \mathrm{b})$ in the reference image in coordinate $(\mathrm{a}, \mathrm{b})$. Finally the pest in the sona massori paddy crop is detected as shown in Figure 4 and Figure 5.By detecting in early stages and taking corrective measures the yield can be improved. 


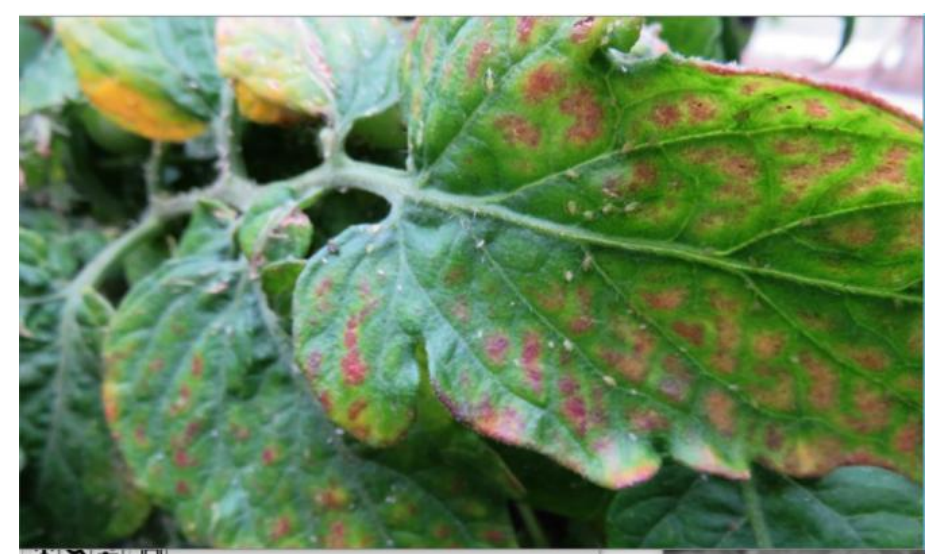

Fig 4: Pest identification

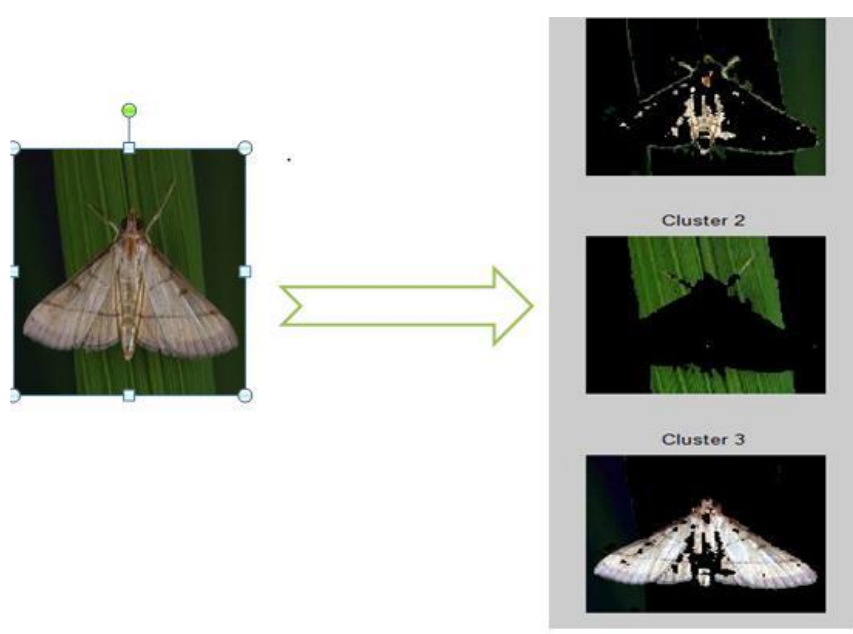

Fig 5: Pest Detection

\section{CONCLUSION}

Image processing techniques plays a vital role in the detection of diseases and pests in sona massori paddy crop plant. Here the first objective is to detect diseases and then detecting the pests which are the main reason for the low yield. Here tilt camera is worn for taking paddy leaves. This proposed system is proved to be reliable for detection of pests. Further economic losses faced by farmers can also be avoided by knowing about the health of the crop and timely examination of the crop. If diseases and pests are detected in the crops at early stage, farmers can take corrective measures to protect the crop.

\section{References}

[1] Arif sait, Massod "Study on Paddy field with Image Processing ", IEEE Conference on Systems, Process and Control, pp. 26-28,2016.

[2] Jackson Peter, "Paddy cultivation and importance of pest control”, JATIT,Vol 09,No.10, pp.113-115, 2016.

[3] Shankar Ganesh and Sara Benjamin, "Paddy insect pests classification based on deep learning," International Journal of Agriculture Science, vol. 1,pp. 12-16, June 2016.

[4] Davoud A, Hossein A, Ali, Mohammad," An Investigation Into Machine Learning Regression Techniques for the Leaf Rust Disease Detection Using Hyperspectral Measurement" 2016 IEEE.

[5] Ravi Dila, Raj Reddy," Detection of diseases on paddy crop Image Processing Techniques" 2015 IEEE International Conference on Computational Networking and Agriculture Research. 
[6] Atlaf Ali,Javed Ali, "A Framework for Stem Diseases in paddy field," IEEE International Conference on agriculture, 2015, Bangladesh.

[7] Santanu Phadikar and Jaya Sil, "Rice Disease Identification using Pattern Recognition", Proceedings of 11th International Conference on Computer and Information Technology (ICCIT 2008) 25-27 December, 2008, Khulna, Bangladesh.

[8] Dr.K.Thangadurai, K.Padmavathi, "Computer Vision image Enhancement for Plant Leaves Disease Detection", 2014 World Congress on Computing and Communication Technologies.

[9] Y. Zhao and X. Li, "Edge-Aware Weighting Enhanced Saliency Segmentation of Pests Images," 2016 International Conference on Computational Science and Computational Intelligence (CSCI), Las Vegas, NV, 2016, pp. 847-851, doi: 10.1109/CSCI.2016.0164.

[10] P. Rajan, B. Radhakrishnan and L. P. Suresh, "Detection and classification of pests from crop images using Support Vector Machine," 2016 International Conference on Emerging Technological Trends (ICETT), Kollam, 2016, pp. 1-6, doi: 10.1109/ICETT.2016.7873750.

[11] A. Singh and M. L. Singh, "Automated color prediction of paddy crop leaf using image processing," 2015 IEEE Technological Innovation in ICT for Agriculture and Rural Development (TIAR), Chennai, 2015, pp. 24-32, doi: 10.1109/TIAR.2015.7358526.

[12] Sindhuja Sankarana, Ashish Mishraa, Reza Ehsania and Cristina Davis, "A Review of Advanced Techniques For Detecting Plant Diseases", Computers and Electronics in Agriculture, vol. 72, pp. 1-13, 2010.

[13] K. H. Choi, S. K. Han, K. Park, K. Kim and S. Kim, "Vision based guidance line extraction for autonomous weed control robot in paddy field," 2015 IEEE International Conference on Robotics and Biomimetics (ROBIO), Zhuhai, 2015, pp. 831-836, doi: 10.1109/ROBIO.2015.7418873.

[14] F. Kugler, D. Schulze, I. Hajnsek, H. Pretzsch and K. P. Papathanassiou, "TanDEM-X Pol-InSAR performance for forest height estimation", Geoscience and Remote Sensing IEEE Transactions on, vol. 52, no. 10, pp. 6404-6422, Oct 2014 